\title{
Effectiveness of hyperbaric oxygen therapy for virus-associated hemorrhagic cystitis after allogeneic hematopoietic stem cell transplantation
}

\author{
Kohei Hosokawa ${ }^{1} \cdot$ Go Aoki $^{1,2}$ - Kinya Ohata ${ }^{1}$. Hiroyuki Takamatsu ${ }^{1} \cdot$ Noriharu Nakagawa $^{1}$. Tatsuya Imi ${ }^{1}$. \\ Noriko Iwaki ${ }^{1} \cdot$ Kiyoaki Ito $^{3} \cdot$ Mitsuhiro Kawano $^{3} \cdot$ Takashi Nakamura $^{4} \cdot$ Masato Takamori $^{5} \cdot$ Ken Ishiyama $^{1}$. \\ Yukio Kondo $^{1}$ · Hirohito Yamazaki ${ }^{1}$ • Shinji Nakao ${ }^{1}$
}

Received: 20 October 2020 / Revised: 2 March 2021 / Accepted: 4 March 2021 / Published online: 16 March 2021

(c) Japanese Society of Hematology 2021

\begin{abstract}
Although some studies have suggested the effectiveness of hyperbaric oxygen (HBO) therapy for hemorrhagic cystitis (HC) after allogeneic hematopoietic stem cell transplantation (HSCT), the role of HBO has not been established. We compared the treatment outcomes of 8 patients with viral $\mathrm{HC}$ (adenovirus [ADV], $n=2$; $\mathrm{BK}$ virus [BKV], $n=6$ ) treated with $\mathrm{HBO}$ $(\mathrm{HBO}[+])$ and 8 patients (ADV, $n=2 ; \mathrm{BKV}, n=6)$ treated with conventional therapy $(\mathrm{HBO}[-])$, such as urinary catheterization and intravenous cidofovir. HBO therapy was performed at 2.1 atmospheres for $90 \mathrm{~min} /$ day until clinical improvement was achieved. The median number of HBO treatments was 10 (range 8-12). The median duration of HBO treatment was 19.5 days (range 10-23 days). All $8 \mathrm{HBO}(+)$ patients achieved complete remission (CR) at a median of 14.5 days (range 5-25 days). Of the $8 \mathrm{HBO}(-)$ patients, 5 (62.5\%) obtained CR and 3 remained symptomatic for 2-6 months. The cumulative incidence of transplant-related mortality at day 100 after allogeneic HSCT was significantly higher in the $\mathrm{HBO}(-)$ patients than in the $\mathrm{HBO}(+)$ patients $(14.2$ vs. $0 \%, P<0.05)$. No severe HBO-related adverse effects were observed. In conclusion, $\mathrm{HBO}$ is a feasible option for treating viral $\mathrm{HC}$ after allogeneic HSCT.
\end{abstract}

Keywords Hyperbaric oxygen therapy $\cdot$ Hemorrhagic cystitis

\section{Introduction}

Kohei Hosokawa and Go Aoki equally contributed to this paper as the first authors.

Shinji Nakao

snakao8205@staff.kanazawa-u.ac.jp

1 Department of Hematology, Kanazawa University Graduate School of Medical Science, 13-1 Takaramachi, Kanazawa, Ishikawa 920-8640, Japan

2 Department of Internal Medicine, Fukui-Ken Saiseikai Hospital, Fukui, Japan

3 Department of Rheumatology, Kanazawa University Graduate School of Medical Science, Kanazawa, Japan

4 Department of Surgery, Jouhoku Hospital, Ishikawa Kinrosha Medical Association, Kanazawa, Japan

5 Asagao Clinic, Hakusan, Japan
Hemorrhagic cystitis (HC) is a common complication after allogeneic hematopoietic stem cell transplantation (alloHSCT). HC occurs in 8.6-24\% of allo-HSCT recipients and severely impairs their quality of life [1-6]. Early-onset HC, which occurs within 1 week after HSCT, mostly develops due to the regimen-related toxicity, while late-onset $\mathrm{HC}$ after engraftment is usually caused by viruses, such as human polyomavirus $\mathrm{BK}(\mathrm{BKV})$, polyomavirus $\mathrm{JC}$, adenovirus (ADV), and cytomegalovirus (CMV). A number of retrospective studies have identified various risk factors for HC after allo-HSCT. These included busulfan (BU)-containing conditioning regimens, unrelated donor, the occurrence of graft-versus-host disease (GVHD), and CMV reactivation [6-9].

Supportive therapies, including forced diuresis, hyperhydration, continuous bladder irrigation, and platelet transfusion, have been the standard of care for HC. Several retrospective studies have reported the efficacy of cidofovir 
(CDV) for BKV-associated HC (BKV-HC) and ADV-associated HC (ADV-HC) after allo-HSCT [10-16]. However, its therapeutic efficacy for CDV has not been comprehensively validated by prospective controlled studies, and the optimal dose remains unclear. In addition, the use of CDV may exacerbate the renal dysfunction that is often associated with HC. The successful intravesical instillation of CDV has been reported, but their clinical efficacy is limited [17].

Hyperbaric oxygen (HBO) therapy is commonly used for the treatment of arterial gas embolism, poorly healing diabetic wounds, osteomyelitis, radiation tissue injury, and carbon monoxide poisoning, and is known to be safe, noninvasive, and cost-effective. HBO has also been shown to ameliorate radiation-induced $\mathrm{HC}$ by promoting fibroblast proliferation and capillary angiogenesis, decreasing edema, and facilitating regeneration of the damaged hypoxic urothelium $[18,19]$. Recent studies indicated the clinical effectiveness of HBO therapy for BKV-HC after allo-HSCT [20-23]. Savva-Bordalo et al. presented 16 patients with $\mathrm{HC}$ after allo-HSCT who underwent HBO therapy, and who showed a high response rate (94\%). However, few studies have compared the clinical effectiveness of HBO therapy with that of conventional therapy in the treatment of $\mathrm{HC}$ after allo-HSCT. In the present study, we analyzed the efficacy and safety of HBO therapy in comparison to conventional therapy for patients with post-HSCT viral HC who were managed in our institution.

\section{Patients and methods}

\section{Patients}

Among the patients who underwent allo-HSCT at our institution between May 2007 and August 2017, 22 patients developed virus-associated $\mathrm{HC}$ and 16 patients without grade II-IV acute GVHD (aGVHD) were included in the study. Their medical records were retrospectively analyzed. Eight of the 16 patients $(50 \%)$ were treated with $\mathrm{HBO}$ therapy $[\mathrm{HBO}(+)]$, while 8 patients $(50 \%)$ who received conventional therapies, such as intravenous hydration with forced diuresis, urinary catheter, and intravenous $\mathrm{CDV}[\mathrm{HBO}(-)]$. The selected treatments for viral $\mathrm{HC}$ were based on the judgment of each individual physician. The clinical characteristics of the patients, including the original diseases, clinical statuses, conditioning regimens, sources of allo-HSCT, and GVHD prophylaxis regimens are shown in Table 1. The median age of the $\mathrm{HBO}(+)$ patients was 51.5 years (range 32-64 years). Antithymocyte globulin (ATG) was administered to 2 (UPNs 3 and 15) of 16 cases as prophylaxis against GVHD. Four (UPNs 3-6) of $8 \mathrm{HBO}(+)$ cases were grafted with peripheral blood stem cells (PBSCs) from an HLAmismatched donor; three (UPNs 4-6) received PBSCs from
HLA-haploidentical donors using post-transplant high-dose cyclophosphamide. The 16 patients received 2-12 (median 12) Gy of total body irradiation. There was no significant difference in the age, original disease, performance status, hematopoietic cell transplant-comorbidity index (HCT$\mathrm{CI}$ ), conditioning regimen, or stem cell source between the $\mathrm{HBO}(+)$ patients and the $\mathrm{HBO}(-)$ patients.

All patients provided their informed consent in accordance with the Declaration of Helsinki. This retrospective analysis was approved by the ethics committee of the Kanazawa University Hospital (No. 2453).

\section{Definitions}

Engraftment was defined as a neutrophil count of $>0.5 \times 10^{9} / \mathrm{L}$ for three consecutive days. HC was diagnosed when microscopic (blood in urine grade $\geq 1+$ ) or macroscopic hematuria accompanied by dysuria, pollakisuria, urinary urgency, and/or the sensation of residual urine, which developed without evidence of bacteriuria [24]. BKVor ADV-HC was diagnosed when a qualitative PCR assay detected $\mathrm{BKV}$ or $\mathrm{ADV}$ in the urine of symptomatic patients. The date of onset of $\mathrm{HC}$ was defined as the first day on which the patient complained of urinary symptoms. The severity of hematuria was graded according to the criteria for adverse effects [25]. Transplant-related mortality (TRM) was defined as death due to any transplantation-related causes, other than disease relapse.

\section{HBO therapy}

HBO therapy was started at 2.1 atmospheres for $90 \mathrm{~min}$ per day from the day on which the patient showed grade II-III symptoms of $\mathrm{HC}$, and therapy was continued until the patient achieved clinical improvement, which was defined as complete resolution (CR) or partial resolution (PR) of macroscopic hematuria and urinary symptoms, such as pain on urination. $\mathrm{CR}$ of $\mathrm{HC}$ was defined as undetectable blood in urine ([-] or $[ \pm]$ for hemoglobin) and the disappearance of dysuria, pollakisuria, urinary urgency, and the sensation of residual urine related to $\mathrm{HC}$.

\section{Statistical analysis}

Fisher's exact test was used for the analysis of categorical variables and the Mann-Whitney's $U$ test was used for the analysis of continuous variables. The Wilcoxon signed-rank test was used for paired samples. The probabilities of TRM were estimated based on the cumulative incidence method to compensate for any competing risks, and the groups were then compared using Gray's test. $P$ values of $<0.05$ were considered to indicate statistical significance. All statistical analyses were performed using the EZR software package 


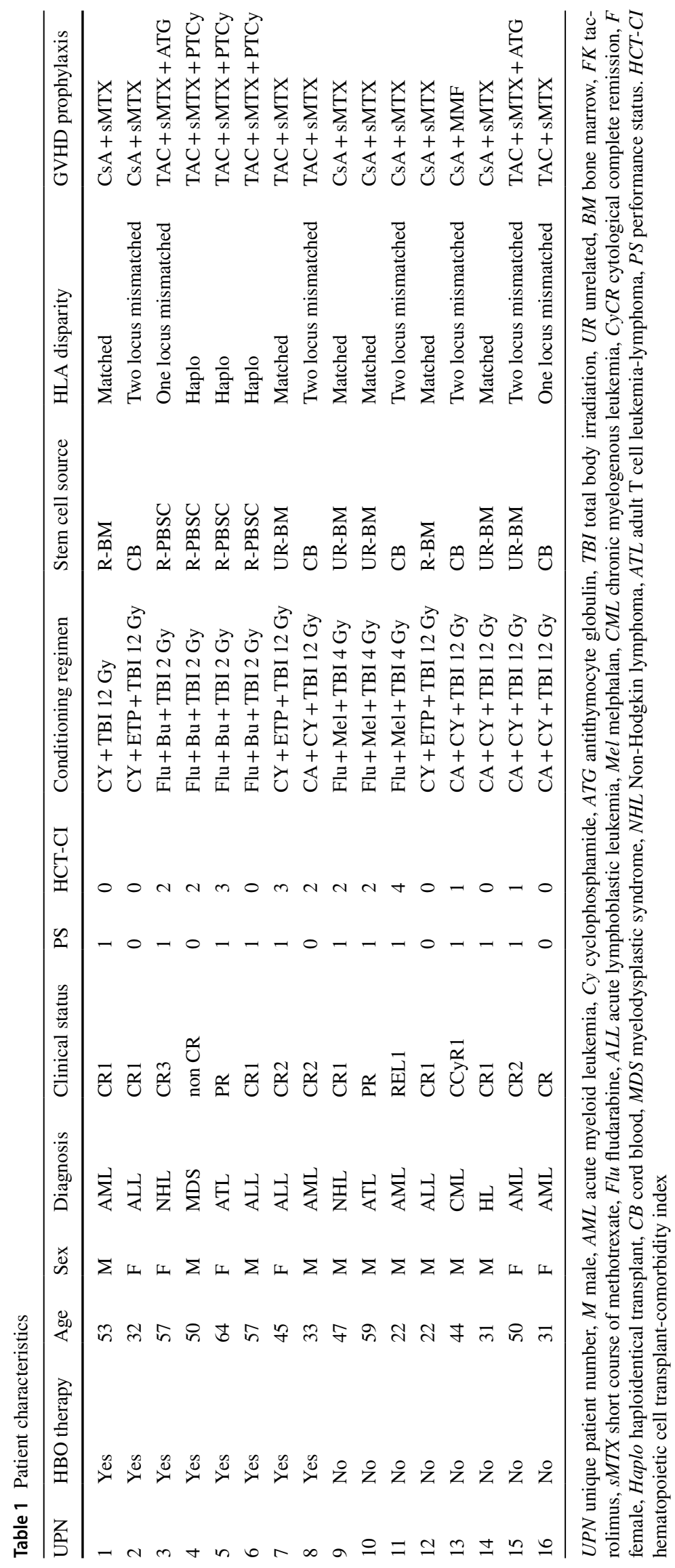


(Saitama Medical Center, Jichi Medical University, Saitama, Japan). Graphs were generated using GraphPad PRISM 8.0 (GraphPad Software, Inc., La Jolla, CA, USA).

\section{Results}

\section{Development of HC}

The 16 patients were diagnosed with virus-associated HC at a median 31 days (range 7-764 days) after allo-HSCT (Table 2). The median time to the onset of HC after alloHSCT in the $\mathrm{HBO}(+)$ patients (median 29 days, range 13-764 days) and $\mathrm{HBO}(-)$ patients (median 48 days, range 7-630 days) did not differ to a statistically significant extent. Among the $8 \mathrm{HBO}(+)$ patients, $2(25 \%$, UPNs 1 and 6$)$ had ADV-HC, while the other 6 (75\%, UPNs 2, 3, 4, 5, 7 and 8) had BKV-HC. Among the $8 \mathrm{HBO}(-)$ patients, $2(25 \%$,
UPNs 9 and 10) and 6 (75\%, UPNs 11-16) had ADV- and BKV-HC, respectively. The severity of $\mathrm{HC}$ was comparable between the $\mathrm{HBO}(+)$ ( 7 had grade II and 1 had grade III) and $\mathrm{HBO}(-)$ ( 7 had grade II and 1 had grade IV) patients.

Three (UPNs 4, 8, and 16) of the 16 patients (19\%) had grade I acute aGVHD; one of these patients received corticosteroids for the treatment of aGVHD at the onset of virusassociated $\mathrm{HC}$ and the patients responded to corticosteroids well. Five of the 16 patients $(31 \%)$ had been treated with ganciclovir (GCV) and/or foscarnet (PFA) for the treatment of CMV antigenemia at the time of the diagnosis of virusassociated HC.

\section{HBO therapy for virus-associated HC}

Table 3 summarizes HBO and conventional therapies, as well as their outcomes. The median number of HBO therapy sessions was 10 (range 8-12) times and the median

Table 2 Clinical characteristics of patients with virus-associated cystitis

\begin{tabular}{|c|c|c|c|c|c|c|c|c|c|}
\hline UPN & Engraftment & $\begin{array}{l}\text { Acute } \\
\text { GVHD }\end{array}$ & Steroid use & $\begin{array}{l}\text { Onset of HC } \\
(\mathrm{d} \text { from } \mathrm{SCT})\end{array}$ & $\begin{array}{l}\text { Hematuria } \\
\text { (Grade) }\end{array}$ & Viruria & Viral infections & Antiviral therapy at $\mathrm{HC}$ & $\begin{array}{l}\text { CR (day } \\
\text { from } \\
\text { therapy) }\end{array}$ \\
\hline 1 & d15 & - & - & 764 & II & ADV & - & - & 25 \\
\hline 2 & d20 & - & - & 24 & II & BKV & - & PFA & 21 \\
\hline 3 & d15 & - & - & 59 & II & BKV & CMV antigenemia & PFA & 13 \\
\hline 4 & d17 & I & + & 38 & II & $\mathrm{BKV}$ & - & - & 13 \\
\hline 5 & $\mathrm{~d} 22$ & - & - & 34 & II & BKV & - & - & 5 \\
\hline 6 & $\mathrm{~d} 18$ & - & - & 18 & II & $\mathrm{ADV}$ & CMV antigenemia & GCV & 11 \\
\hline 7 & $\mathrm{~d} 17$ & - & - & 13 & II & $\mathrm{BKV}$ & $\mathrm{CMV}$ antigenemia & PFA & 22 \\
\hline 8 & $\mathrm{~d} 18$ & I & - & 21 & III & BKV & - & - & 16 \\
\hline 9 & d17 & - & - & 630 & II & $\mathrm{ADV}$ & - & - & 24 \\
\hline 10 & $\mathrm{~d} 17$ & - & - & 21 & II & ADV & - & - & 17 \\
\hline 11 & d16 & - & - & 22 & II & $\mathrm{BKV}$ & HHV-6 & PFA & 25 \\
\hline 12 & $\mathrm{~d} 25$ & - & - & 7 & II & BKV & - & - & 13 \\
\hline 13 & d19 & - & - & 68 & II & BKV & $\mathrm{CMV}$ antigenemia & GCV, PFA & $\mathrm{NE}$ \\
\hline 14 & $\mathrm{~d} 21$ & - & - & 28 & IV & $\mathrm{BKV}$ & - & Ara-A & $\mathrm{NE}$ \\
\hline 15 & d17 & - & - & 96 & II & $\mathrm{BKV}$ & $\mathrm{CMV}$ antigenemia & PFA, DLI, CDV & $\mathrm{NE}$ \\
\hline 16 & $\mathrm{~d} 27$ & I & - & 200 & II & BKV & - & - & 216 \\
\hline
\end{tabular}

$S C T$ stem cell transplantation, $E B V$ Epstein-Barr virus, $C M V$ Cytomegalovirus, $G C V$ ganciclovir, $P F A$ foscarnet, $C D V$ cidofovir, $D L I$ donor lymphocyte infusion, $C R$ complete resolution, GVHD graft-versus-host disease, $N E$ not evaluable

Table 3 Summary of the treatment of patients with virusassociated cystitis

\begin{tabular}{lllll}
\hline & & HBO+group $(n=8)$ & HBO- group $(n=8)$ & \\
\hline Onset of HC & & $29(13-764)$ & $48(7-630)$ & n.s \\
Virus & BKV & $6(75 \%)$ & $6(75 \%)$ & n.s \\
& ADV & $2(25 \%)$ & $2(25 \%)$ & n.s \\
Number of HBO therapy & Median (range) & $10(8-12)$ & NA & \\
From therapy to CR & Median (range) & $14.5(5-25)$ & $24(13-216)$ & n.s \\
Response rate & & $8 / 8(100 \%)$ & $5 / 8(62.5 \%)$ & n.s \\
\hline
\end{tabular}

$H C$ hemorrhagic cystitis, $C R$ complete resolution, $N A$ not applicable, $n . s$. not significant 
duration of HBO therapy was 19.5 days (range 10-23 days). The response rate was $100 \%(8 / 8)$ in $\mathrm{HBO}(+)$ patients and $62.5 \%(5 / 8)$ in $\mathrm{HBO}(-)$ patients. All eight $\mathrm{HBO}(+)$ patients achieved a PR in 2-17 (median 6.5) days and a CR in 5-25 (median 14.5) days after the start of HBO therapy. The time from therapy to $\mathrm{CR}$ in $\mathrm{HBO}(+)$ patients tended to be shorter than that in $\mathrm{HBO}(-)$ patients (median 14.5 vs. 24 days), but it was not statistically significant. The urine virus load decreased 2 weeks after the start of HBO therapy in one patient (UPN2), which we previously reported [22].

Of $8 \mathrm{HBO}(-)$ patients, 3 (37.5\%, UPNs 13-15) did not achieve a PR or CR and remained symptomatic for 2-6 months.

\section{Safety and tolerability of treatment}

There were no severe adverse effects due to HBO therapy in any of the $8 \mathrm{HBO}(+)$ patients. One patient (UPN 4) had unilateral serous otitis media after HBO therapy, which was resolved with tympanostomy. Although an ear pain or discomfort and middle ear barotrauma (MEB) [26] are common adverse effects of $\mathrm{HBO}$, none of the other $7 \mathrm{HBO}(+)$ patients developed these complications.

One (UPN 14) of the $\mathrm{HBO}(-)$ patients with BKV-HC was complicated by renal dysfunction due to clot retention in the ureters and eventually died of uremia. None of the $8 \mathrm{HBO}(+)$ patients and none of the other $7 \mathrm{HBO}(-)$ patients developed renal functional impairment during the course of $\mathrm{HC}$.

\section{Transplant outcomes}

All $8 \mathrm{HBO}(+)$ patients survived more than 100 days after HSCT. Two HBO(+) patients (UPNs 1 and 5) died of relapse more than 1 years after HSCT, but there was no transplantrelated mortality (TRM). Of $4 \mathrm{HBO}(-)$ patients who died 2-17 months after allo-HSCT, 3 (UPNs 13-15) succumbed to TRM, which was associated with infection and organ failure. The other $\mathrm{HBO}(-)$ patient (UPN 11) died of relapse more than 1 years after HSCT. The cumulative incidence of TRM at day 100 after allo-HSCT was significantly higher in $\mathrm{HBO}(-)$ patients than in $\mathrm{HBO}(+)$ patients (14.2 vs. $0 \%$, $P<0.05$, Fig. 1).

\section{Discussion}

This retrospective study revealed several potential benefits of HBO therapy in the treatment of $\mathrm{HC}$ after allo-HSCT. HBO therapy produced a higher CR rate (100\%) and shortened the time from therapy to $\mathrm{CR}$ in comparison to conventional therapies. In addition, the TRM rate at day 100 in $\mathrm{HBO}(+)$ patients was lower than that in $\mathrm{HBO}(-)$ patients, leading to a higher non-relapse survival rate in $\mathrm{HBO}(+)$ patients.
Figure 1 Hosokawa, et al

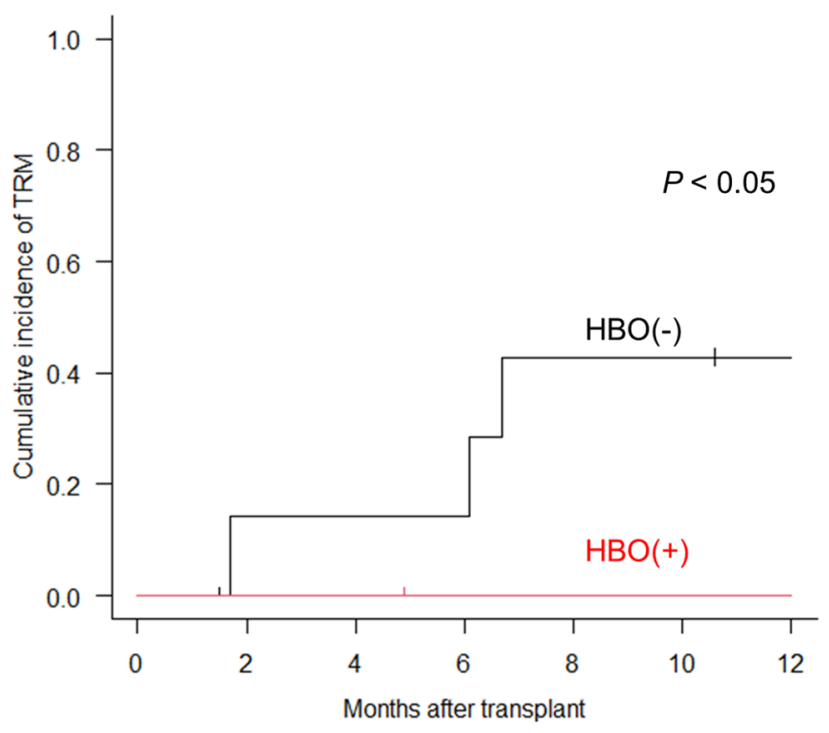

Fig. 1 The cumulative incidence of transplant related mortality in the patients with or without HBO therapy. The cumulative incidence of transplant-related mortality (TRM) in the patients with or without HBO therapy for virus-associated hemorrhagic cystitis after allogeneic HSCT. The cumulative incidence of TRM at day 100 was significantly higher in the $\mathrm{HBO}(-)$ patients than in the $\mathrm{HBO}(+)$ patients (14.2 vs. $0 \%, P<0.05$ )

All eight patients completed HBO therapy without severe adverse effects, with success even in patients receiving corticosteroids for the treatment of aGVHD. HBO therapy is, therefore, thought to be feasible option for the treatment of virus-associated $\mathrm{HC}$ after allo-HSCT.

Several studies have demonstrated the effectiveness of HBO therapy for BKV-HC [20-23]. A retrospective study from Portugal showed that 15 (94\%) of $16 \mathrm{BKV}-\mathrm{HC}$ patients treated with HBO therapy achieved a CR after a median of 17 days (range 4-116 days) [20]. In a pediatric cohort of 10 children with BKV-HC, HBO therapy produced a CR in 9 (90\%) patients after a median of 15 days (range 10-37 days) [21]. Another retrospective study reported that all 5 adult patients transplanted from matched unrelated donors responded to HBO therapy and achieved a CR after the mean of 13 sessions (range 11-30 sessions). However, none of these studies directly compared the treatment outcomes between patients who received HBO and conventional therapy at a single institution. We showed that the time from therapy to $\mathrm{CR}$ in $\mathrm{HBO}(+)$ patients was significantly shorter than that in $\mathrm{HBO}(-)$ patients. Although the number of patients was limited, our comparative study provided further evidence that $\mathrm{HBO}$ therapy is a promising treatment option for patients with HC after allo-HSCT.

To our knowledge, there are no standard treatment guidelines for post-HSCT HC. Intravenous hydration with forced 
diuresis is usually conducted; however, this is a supportive treatment that does not show reliable efficacy. The efficacy of CDV against ADV-HC has been demonstrated by a prospective clinical trial [12]; however, the role of CDV in the treatment of BKV-HC was only investigated in retrospective studies [14, 27, 28]. For example, in a report from the European Group for Blood and Marrow Transplantation (EBMT), intravenous or intravesical CDV was administered to 62 patients with BKV-HC [14], and 41 (66\%) achieved CR and 8 (13\%) achieved a PR. Notably, of the 57 patients who received intravenous CDV treatment, 17 (30\%) experienced renal dysfunction. We did not use CDV for the treatment of our patients with $\mathrm{HC}$, with the exception of one patient (UPN15) with severe acute respiratory distress syndrome caused by pneumonia, because CDV has not received approval in Japan. Given the nephrotoxicity of intravenous CDV and the safety profile of HBO therapy, it may be a reasonable approach to start treatment with HBO in the early course of $\mathrm{HC}$, particularly for patients with renal dysfunction.

HBO has been utilized as primary or adjunctive therapy for several medical conditions where tissue damage is triggered by hypoxic injury, due to its stimulatory effects on fibroblast proliferation, angiogenesis, and wound healing [29]. Although the precise pathogenesis of virus-associated $\mathrm{HC}$ remains unclear, virus-infected urothelial cells may be associated with the denudation of the damaged mucosa in patients with $\mathrm{HC}$ [30]. HBO therapy may protect urothelial cells by stimulating fibroblast proliferation, angiogenesis, and wound healing.

The present study was associated with some limitations. The study population was relatively small and the patients were treated at a single center; thus, there may be some bias in relation to the treatment outcomes. Although the urine BKV load markedly decreased 2 weeks after the start of HBO therapy in UPN2, we could not monitor the change of the viral load in the other 15 patients. The impact of HBO therapy on $\mathrm{HC}$ needs to be determined in a prospective study where the viral load is sequentially followed in every sample.

In conclusion, our comparative study provided further evidence that $\mathrm{HBO}$ is a feasible option for the treatment of viral $\mathrm{HC}$ after allogeneic HSCT.

Acknowledgements We thank the inpatient nursing team and the support staff for the excellent care they provided to our donors, patients and their families.

Author contributions $\mathrm{KH}, \mathrm{GA}$, and SN participated in the design of this analysis. KH, GA, KO, HT, NN, TM, NI, KI, YK, and HY recruited patients to participate in the study. $\mathrm{KI}$ and $\mathrm{MK}$ performed the qPCR assays. TN and MT performed the HBO therapy. KH and GA analyzed the data. $\mathrm{KH}, \mathrm{GA}$, and $\mathrm{SN}$ wrote the manuscript. All the authors critically reviewed the manuscript content and agreed with the submission of the final manuscript. $\mathrm{KH}$ and GA contributed equally to this work.

\section{Declarations}

Conflict of interest The authors declare no conflicts of interest in association with the present study.

\section{References}

1. Miyamura K, Takeyama K, Kojima S, Minami S, Matsuyama K, Morishima Y, et al. Hemorrhagic cystitis associated with urinary excretion of adenovirus type 11 following allogeneic bone marrow transplantation. Bone Marrow Transplant. 1989;4(5):533-5.

2. Akiyama H, Kurosu T, Sakashita C, Inoue T, Mori S, Ohashi $\mathrm{K}$, et al. Adenovirus is a key pathogen in hemorrhagic cystitis associated with bone marrow transplantation. Clin Infect Dis. 2001;32(9):1325-30.

3. Asano Y, Kanda Y, Ogawa N, Sakata-Yanagimoto M, Nakagawa M, Kawazu M, et al. Male predominance among Japanese adult patients with late-onset hemorrhagic cystitis after hematopoietic stem cell transplantation. Bone Marrow Transplant. 2003;32(12):1175-9.

4. Yamamoto R, Kusumi E, Kami M, Yuji K, Hamaki T, Saito A, et al. Late hemorrhagic cystitis after reduced-intensity hematopoietic stem cell transplantation (RIST). Bone Marrow Transplant. 2003;32(11):1089-95.

5. Nakazawa Y, Saito S, Yanagisawa R, Suzuki T, Ito T, Ishida F, et al. Recipient seropositivity for adenovirus type 11 (AdV11) is a highly predictive factor for the development of AdV11-induced hemorrhagic cystitis after allogeneic hematopoietic SCT. Bone Marrow Transplant. 2013;48(5):737-9.

6. Mori Y, Miyamoto T, Kato K, Kamezaki K, Kuriyama T, Oku S, et al. Different risk factors related to adenovirus- or BK virus-associated hemorrhagic cystitis following allogeneic stem cell transplantation. Biol Blood Marrow Transplant. 2012;18(3):458-65.

7. Arai Y, Maeda T, Sugiura H, Matsui H, Jo T, Ueda T, et al. Risk factors for and prognosis of hemorrhagic cystitis after allogeneic stem cell transplantation: retrospective analysis in a single institution. Hematology. 2012;17(4):207-14.

8. Hayden RT, Gu Z, Liu W, Lovins R, Kasow K, Woodard P, et al. Risk factors for hemorrhagic cystitis in pediatric allogeneic hematopoietic stem cell transplant recipients. Transpl Infect Dis. 2015;17(2):234-41.

9. Lunde LE, Dasaraju S, Cao Q, Cohn CS, Reding M, Bejanyan $\mathrm{N}$, et al. Hemorrhagic cystitis after allogeneic hematopoietic cell transplantation: risk factors, graft source and survival. Bone Marrow Transplant. 2015;50(11):1432-7.

10. Hoffman JA, Shah AJ, Ross LA, Kapoor N. Adenoviral infections and a prospective trial of cidofovir in pediatric hematopoietic stem cell transplantation. Biol Blood Marrow Transplant. 2001;7(7):388-94.

11. Ljungman P, Ribaud P, Eyrich M, Matthes-Martin S, Einsele H, Bleakley $\mathrm{M}$, et al. Cidofovir for adenovirus infections after allogeneic hematopoietic stem cell transplantation: a survey by the Infectious Diseases Working Party of the European Group for Blood and Marrow Transplantation. Bone Marrow Transplant. 2003;31(6):481-6.

12. Nagafuji K, Aoki K, Henzan H, Kato K, Miyamoto T, Eto T, et al. Cidofovir for treating adenoviral hemorrhagic cystitis in hematopoietic stem cell transplant recipients. Bone Marrow Transplant. 2004;34(10):909-14.

13. Yusuf U, Hale GA, Carr J, Gu Z, Benaim E, Woodard P, et al. Cidofovir for the treatment of adenoviral infection in pediatric 
hematopoietic stem cell transplant patients. Transplantation. 2006;81(10):1398-404.

14. Cesaro S, Hirsch HH, Faraci M, Owoc-Lempach J, Beltrame A, Tendas A, et al. Cidofovir for BK virus-associated hemorrhagic cystitis: a retrospective study. Clin Infect Dis. 2009;49(2):233-40.

15. Savona MR, Newton D, Frame D, Levine JE, Mineishi S, Kaul DR. Low-dose cidofovir treatment of BK virus-associated hemorrhagic cystitis in recipients of hematopoietic stem cell transplant. Bone Marrow Transplant. 2007;39(12):783-7.

16. Philippe M, Ranchon F, Gilis L, Schwiertz V, Vantard N, Ader F, et al. Cidofovir in the Treatment of BK Virus-Associated Hemorrhagic Cystitis after Allogeneic Hematopoietic Stem Cell Transplantation. Biol Blood Marrow Transplant. 2016;22(4):723-30.

17. Sakurada M, Kondo T, Umeda M, Kawabata H, Yamashita K, Takaori-Kondo A. Successful treatment with intravesical cidofovir for virus-associated hemorrhagic cystitis after allogeneic hematopoietic stem cell transplantation: a case report and a review of the literature. J Infect Chemother. 2016;22(7):495-500.

18. Yoshida T, Kawashima A, Ujike T, Uemura M, Nishimura K, Miyoshi S. Hyperbaric oxygen therapy for radiation-induced hemorrhagic cystitis. Int J Urol. 2008;15(7):639-41.

19. Chong KT, Hampson NB, Corman JM. Early hyperbaric oxygen therapy improves outcome for radiation-induced hemorrhagic cystitis. Urology. 2005;65(4):649-53.

20. Savva-Bordalo J, Pinho Vaz C, Sousa M, Branca R, Campilho $\mathrm{F}$, Resende R, et al. Clinical effectiveness of hyperbaric oxygen therapy for BK-virus-associated hemorrhagic cystitis after allogeneic bone marrow transplantation. Bone Marrow Transplant. 2012;47(8):1095-8.

21. Zama D, Masetti R, Vendemini F, Di Donato F, Morelli A, Prete A, et al. Clinical effectiveness of early treatment with hyperbaric oxygen therapy for severe late-onset hemorrhagic cystitis after hematopoietic stem cell transplantation in pediatric patients. Pediatr Transplant. 2013;17(1):86-91.

22. Hosokawa K, Yamazaki H, Nakamura T, Yoroidaka T, Imi T, Shima Y, et al. Successful hyperbaric oxygen therapy for refractory BK virus-associated hemorrhagic cystitis after cord blood transplantation. Transpl Infect Dis. 2014;16(5):843-6.
23. Urbaniak-Kujda D, Kapelko-Slowik K, Biernat M, Dybko J, Laszkowska M, Kuliczkowski K. The use of hyperbaric oxygen therapy in the treatment of hemorrhagic cystitis after allogeneic stem cell transplantation from an unrelated donor. Int $\mathrm{J}$ Hematol. 2015;102(3):364-7.

24. Kloos RQ, Boelens JJ, de Jong TP, Versluys B, Bierings M. Hemorrhagic cystitis in a cohort of pediatric transplantations: incidence, treatment, outcome, and risk factors. Biol Blood Marrow Transplant. 2013;19(8):1263-6.

25. Droller MJ, Gomolka D. Expression of the cellular immune response during tumor development in an animal model of bladder cancer. J Urol. 1982;128(6):1385-9.

26. Heyboer M 3rd, Sharma D, Santiago W, McCulloch N. Hyperbaric oxygen therapy: side effects defined and quantified. Adv Wound Care (New Rochelle). 2017;6(6):210-24.

27. Kwon HJ, Kang JH, Lee JW, Chung NG, Kim HK, Cho B. Treatment of BK virus-associated hemorrhagic cystitis in pediatric hematopoietic stem cell transplant recipients with cidofovir: a single-center experience. Transpl Infect Dis. 2013;15(6):569-74.

28. Gorczynska E, Turkiewicz D, Rybka K, Toporski J, Kalwak K, Dyla A, et al. Incidence, clinical outcome, and management of virus-induced hemorrhagic cystitis in children and adolescents after allogeneic hematopoietic cell transplantation. Biol Blood Marrow Transplant. 2005;11(10):797-804.

29. Tibbles PM, Edelsberg JS. Hyperbaric-oxygen therapy. N Engl J Med. 1996;334(25):1642-8.

30. Li R, Sharma BN, Linder S, Gutteberg TJ, Hirsch HH, Rinaldo $\mathrm{CH}$. Characteristics of polyomavirus BK (BKPyV) infection in primary human urothelial cells. Virology. 2013;440(1):41-50.

Publisher's Note Springer Nature remains neutral with regard to jurisdictional claims in published maps and institutional affiliations. 